\title{
Genome-wide association study of individual sugar content in fruit of Japanese pear (Pyrus spp.)
}

Sogo Nishio ${ }^{1 *}$, Takeshi Hayashi², Kenta Shirasawa ${ }^{3}$, Toshihiro Saito ${ }^{1}$, Shingo Terakami ${ }^{1}$, Norio Takada ${ }^{1}$, Yukie Takeuchi ${ }^{1}$, Shigeki Moriya ${ }^{4}$ and Akihiko Itai $^{5}$

\begin{abstract}
Background: Understanding mechanisms of sugar accumulation and composition is essential to determining fruit quality and maintaining a desirable balance of sugars in plant storage organs. The major sugars in mature Rosaceae fruits are sucrose, fructose, glucose, and sorbitol. Among these, sucrose and fructose have high sweetness, whereas glucose and sorbitol have low sweetness. Japanese pear has extensive variation in individual sugar contents in mature fruit. Increasing total sugar content and that of individual high-sweetness sugars is a major target of breeding programs. The objective of this study was to identify quantitative trait loci (QTLS) associated with fruit traits including individual sugar accumulation, to infer the candidate genes underlying the QTLs, and to assess the potential of genomic selection for breeding pear fruit traits.
\end{abstract}

Results: We evaluated 10 fruit traits and conducted genome-wide association studies (GWAS) for 106 cultivars and 17 breeding populations (1112 F1 individuals) using 3484 tag single-nucleotide polymorphisms (SNPs). By implementing a mixed linear model and a Bayesian multiple-QTL model in GWAS, 56 SNPs associated with fruit traits were identified. In particular, a SNP located close to acid invertase gene PPAIV3 on chromosome 7 and a newly identified SNP on chromosome 11 had quite large effects on accumulation of sucrose and glucose, respectively. We used 'Golden Delicious' doubled haploid 13 (GDDH13), an apple reference genome, to infer the candidate genes for the identified SNPs. In the region flanking the SNP on chromosome 11, there is a tandem repeat of early responsive to dehydration (ERD6)-like sugar transporter genes that might play a role in the phenotypes observed.

Conclusions: SNPs associated with individual sugar accumulation were newly identified at several loci, and candidate genes underlying QTLs were inferred using advanced apple genome information. The candidate genes for the QTLs are conserved across Pyrinae genomes, which will be useful for further fruit quality studies in Rosaceae. The accuracies of genomic selection for sucrose, fructose, and glucose with genomic best linear unbiased prediction (GBLUP) were relatively high (0.67-0.75), suggesting that it would be possible to select individuals having high-sweetness fruit with high sucrose and fructose contents and low glucose content.

Keywords: Pyrus pyrifolia Nakai, Acid invertase, ERD6-like sugar transporter, Apple, Genome selection

*Correspondence: nishios@affrc.go.jp

1 Institute of Fruit Tree and Tea Science, NARO (NIFTS), 2-1 Fujimoto,

Tsukuba, Ibaraki 305-8605, Japan

Full list of author information is available at the end of the article

\section{Background}

Pears (Pyrus spp.) belong to the subtribe Pyrinae of the Rosaceae and are one of the most important fruit crops in temperate regions. The origin of pear is presumed to be in the mountainous regions of southwestern China and to date back to the Tertiary period ( 65 to 55 million years 
ago) [1]. Pears that dispersed toward the west became domesticated as European pear (P. communis L.), whereas others that dispersed toward the east gave rise to Asian pear species. Asian pears have fruit with crisp, juicy, and sandy texture that is edible just after harvesting. Records of pear cultivation in China have been found from 2000 to 3300 years ago $[1,2]$, and major cultivated Asian pears are traditionally classified into three species: $P$. ussuriensis Maxim., $P$. bretschneideri Rehder, and $P$. pyrifolia (Burm. f.) Nakai [3, 4]. Among the three species, P. pyrifolia is presumed to have been introduced into Japan prehistorically and became the major species in Japan [5]. Previous reports suggested that there were opportunities for ancient cultivar exchange between Japan and eastern China [6, 7], but the varieties currently cultivated in Japan and China are genetically different from each other, suggesting that they have different breeding histories. In Japan, local cultivar 'Nijisseiki' has been one of the leading cultivars, and 'Nijisseiki' and its relatives have been repeatedly used as parents in breeding programs, suggesting that recent cultivars have narrow genetic diversity [7].

Some fruit traits that are important for pear breeding programs and have been genetically studied are fruit harvesting day, fruit weight, fruit hardness, acid content, and sweetness [8-11]. Among these, sweetness is the most important factor determining fruit quality [9]. Fruit sweetness is controlled not only by the total sugar content but also by individual sugar composition. While sucrose is the major individual sugar in carbohydrate translocation from source to sink in most crops, the Rosaceae are unique in that sorbitol plays an important role in this process [12]. After the sugar loaded into Rosaceae fruit is converted by several enzymes that play a critical role in sugar metabolism during fruit development [12-17], sucrose, fructose, glucose, and sorbitol accumulate in mature Rosaceae fruit. These sugars have different levels of sweetness per unit mass $(\mathrm{g})$ : if sucrose is rated 1 , then fructose is $1.50-1.75$, glucose is $0.70-$ 0.80 , and sorbitol is $0.55-0.70$ [18-20]. The mechanisms of sugar accumulation and interconversion are important not only in fruit but also in plant storage organs such as sugar beet taproots, sugarcane stems, and potato tubers [21-23]. Whereas increasing sucrose yield and concentration are important breeding objectives in sugar beet and sugarcane, the content of the reducing sugars fructose and glucose during storage affects the culinary quality of potato products such as chips and French fries.

In a study of various Rosaceae species, pear had a large variation in individual sugar contents in mature fruit [24], whereas cultivar collections of apple and peach had less variation [15, 25-27]. Fructose is dominant in the fruit of most apple cultivars [25, 26], while sucrose is dominant in most peach cultivars [15, 27]. QTLs associated with the conversion of sucrose to hexose in mature fruit were identified on chromosomes 1 and 7 in Japanese pear [9]. Simple sequence repeats (SSRs) corresponding to the regions flanking acid invertase genes PPAIV3 and PPAIV1 were detected within the QTL intervals. The enzymes encoded by these genes are located in the vacuole, where they catalyze the conversion of sucrose to hexose. Large-effect QTLs that control the conversion of sucrose to hexose were also identified at the similar position on apple chromosome 1 [28]. Moreover, QTLs for soluble solids concentration (SSC) have been mapped on pear chromosomes $2,4,5,6$, and $8[11,29]$, though the effects of these QTLs fluctuated from year to year.

Whole-genome duplications are suggested to have occurred in pear and apple, as their genome sequences have extensive syntenic blocks covering much of the chromosomes $(2 n=2 x=34)$ [30]. In addition to synteny of their whole genomes, these species also have interesting genes and QTLs in common. The $S$ haplotypes that control gametophytic self-incompatibility [31] and genes for susceptibility to Alternaria alternata [32, 33] are located at the same positions in both genomes. A member of the 1-aminocyclopropane-1-carboxylate synthase (ACS) gene family is related to several important fruit traits including fruit harvesting day, storage ability, and fruit drop [11, 34-36]. QTLs for harvesting date on chromosome 3 were commonly identified in several studies [11, 37-39]. Because of the high similarity between Pyrus and Malus genome sequences, pear genetic studies have been conducted using advanced apple genome information $[9,40]$. The genome of a doubled haploid 'Golden Delicious' (GDDH13) composed of 280 assembled scaffolds and arranged into 17 pseudomolecules is now the most widely used reference genome in apple genetic studies [41]. Although draft genomes of Chinese pear, wild pear in China (P. betulaefolia), and European pear are available [42-44], a draft genome of Japanese pear has not yet been available.

Several useful DNA markers have been developed and applied in Japanese pear breeding programs: these include DNA markers to identify self-compatibility [45, 46], a molecular marker associated with disease resistance genes $[47,48]$, and markers associated with fruit harvesting day $[8,9,11]$. Unlike some traits controlled by a single gene or large-effect QTLs, marker-assisted selection (MAS) for traits controlled by multiple minor genes has not been applied in pear breeding programs. Currently, genomic selection (GS) is gaining attention as an efficient breeding method for such traits in fruit trees. GS utilizes predicted breeding values given by prediction models based on genome-wide single-nucleotide polymorphism (SNP) data to enable selection of superior 
individuals. The potential of GS for use in Japanese pear breeding was assessed by Minamikawa et al. [10], who used 86 varieties (84 Japanese pear, 2 Chinese pear) and 765 F1 trees from 16 breeding populations (full-sib families) genotyped for SNPs to compare the accuracy of genomic prediction obtained using 12 different methods. The mean prediction accuracy for these models was about 0.6 for fruit quality traits, but those for physiological disorders such as heart rot and watercore were low.

In this study, we updated Minamikawa et al.s [10] study by further evaluating sugar components, by increasing the numbers of cultivars and individuals, and by applying a more powerful genotyping method. Here, we used double-digest restriction-site associated DNA sequencing (ddRAD-Seq) to genotype 106 cultivars and 17 breeding populations (1112 F1 individuals), representing about $40 \%$ more genotypes than in the previous study. This method uses two restriction enzymes, which provides an advantage in precise and repeatable selection and enables paired-end sequencing of identical loci across multiple samples using next-generation sequencing, reducing the cost and time for genotyping [49]. To characterize the sugar metabolism in these materials and to eventually increase the content of specific sugars, here we measured individual sugar contents as well as total sugar content. The objective of this study was to identify the QTLs associated with individual sugar contents and some fruit traits, and to infer the candidate genes for these QTLs. Because many important genes and QTLs have been identified in the apple genome, we used it as a reference genome to identify the chromosome positions of QTLs and surrounding genes. We also examined the potential of GS and considered strategies for introducing GS into pear breeding programs. The genotypes and phenotypes obtained would be a source of information for both practical pear breeding programs and GS.

\section{Results}

\section{Phenotypic distribution of individual sugars}

The average contents of sucrose (SUC), fructose (FRU), glucose (GLC), sorbitol (SOR), and total sugar content (TSC) averaged over 1218 individuals (Table 1) were $43.1,40.6,14.9,32.2$, and $130.7 \mathrm{mg} / \mathrm{ml}$, respectively. SUC showed the greatest range of phenotypic variation (1.6$117.0 \mathrm{mg} / \mathrm{ml}$, variance 355.6; Table S1). The distributions of FRU, GLC, SOR, and TSC were much narrower: 9.6-70.7 $\mathrm{mg} / \mathrm{ml}$ (variance 91.9), $0-35.0 \mathrm{mg} / \mathrm{ml}$ (variance 64.3 ), $11.5-56.3 \mathrm{mg} / \mathrm{ml}$ (variance 56.5 ), and 101.3 $173.8 \mathrm{mg} / \mathrm{ml}$ (variance 125.2), respectively. Fruit harvest time (HarT) ranged over 93 days (July 23 to October 23, mean August 31; Table S1). The range of fruit weight (FruW) was 92.5-1016.1 g (mean 394.2 g), fruit hardness

Table 1 Populations and cultivars used in this study and the proportion of genetic clusters at $K=4$

\begin{tabular}{|c|c|c|c|c|c|c|c|}
\hline Population ID & Seed parent & Pollen parent & $\begin{array}{l}\text { Number of } \\
\text { individuals }\end{array}$ & "orange" cluster & $\begin{array}{l}\text { "light blue" } \\
\text { cluster }\end{array}$ & $\begin{array}{l}\text { "dark blue" } \\
\text { cluster }\end{array}$ & "green” cluster \\
\hline 523 & Akizuki & $373-55$ & 106 & 0.00 & 0.00 & 1.00 & 0.00 \\
\hline 538 & Akizuki & Hoshiakari & 28 & 0.00 & 0.66 & 0.34 & 0.00 \\
\hline 539 & Akizuki & $450-63$ & 62 & 0.00 & 0.01 & 0.99 & 0.00 \\
\hline 541 & Kosui & Hoshiakari & 47 & 0.00 & 0.68 & 0.26 & 0.05 \\
\hline 542 & Akiakari & $450-7$ & 33 & 0.00 & 0.30 & 0.40 & 0.29 \\
\hline 543 & Natsushizuku & Hoshiakari & 78 & 0.00 & 0.71 & 0.29 & 0.00 \\
\hline 545 & Kosui & Inagi & 38 & 0.04 & 0.04 & 0.44 & 0.49 \\
\hline 546 & Akizuki & Akiakari & 36 & 0.00 & 0.19 & 0.54 & 0.27 \\
\hline 547 & Akiakari & Okuroku & 70 & 0.00 & 0.02 & 0.03 & 0.95 \\
\hline 574 & $450-63$ & Chikusui & 51 & 0.00 & 0.03 & 0.97 & 0.00 \\
\hline 578 & Hoshiakari & Narumi & 100 & 0.00 & 0.99 & 0.01 & 0.00 \\
\hline 581 & Kanta & Hoshiakari & 62 & 0.51 & 0.49 & 0.00 & 0.00 \\
\hline 588 & Hoshiakari & Tsukuba49 & 121 & 0.00 & 0.99 & 0.01 & 0.00 \\
\hline 589 & Kanta & Rinka & 49 & 0.77 & 0.07 & 0.04 & 0.12 \\
\hline 590 & $373-055$ & Kanta & 39 & 0.56 & 0.00 & 0.44 & 0.00 \\
\hline 591 & Tsukuba51 & Kanta & 74 & 0.99 & 0.00 & 0.00 & 0.00 \\
\hline 592 & Shurei & Kanta & 72 & 0.61 & 0.00 & 0.39 & 0.00 \\
\hline 595 & Osa gold & Chikusui & 46 & 0.12 & 0.02 & 0.68 & 0.18 \\
\hline \multicolumn{3}{|c|}{ Cultivar collection } & 106 & 0.13 & 0.14 & 0.37 & 0.36 \\
\hline
\end{tabular}

The breeding populations are the product of about five to seven generations of crossing in a pear breeding program at the Institute of Fruit Tree and Tea Science Population structure was estimated for each individuals in software ADMIXTURE $1.30 \mathrm{~K}=4$ 


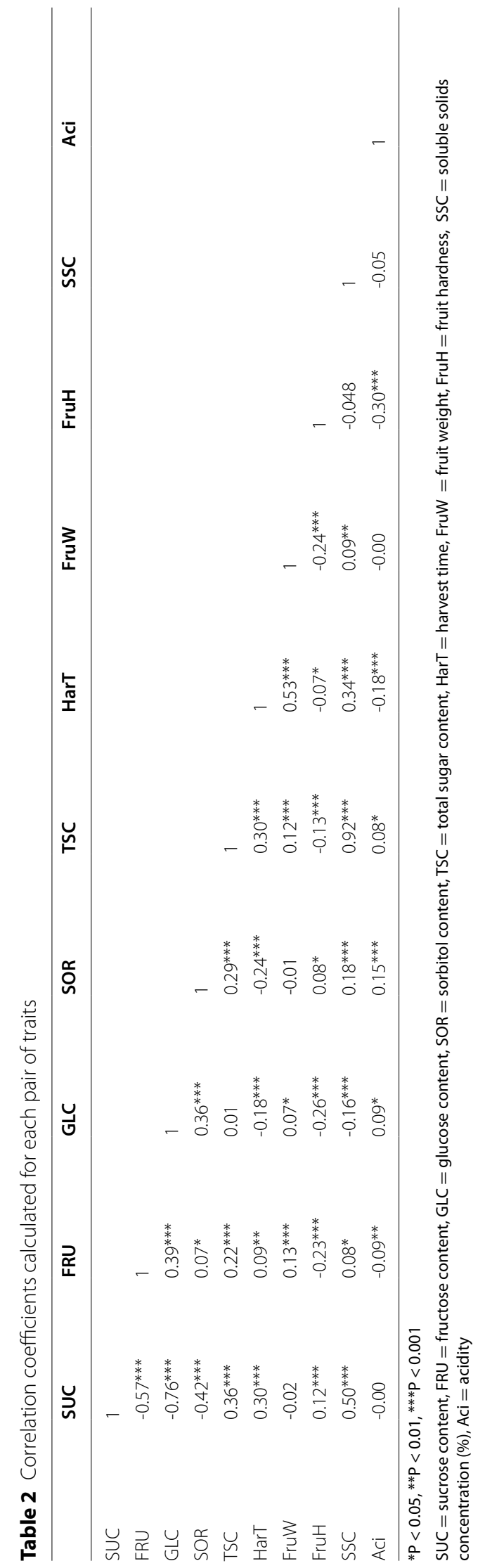




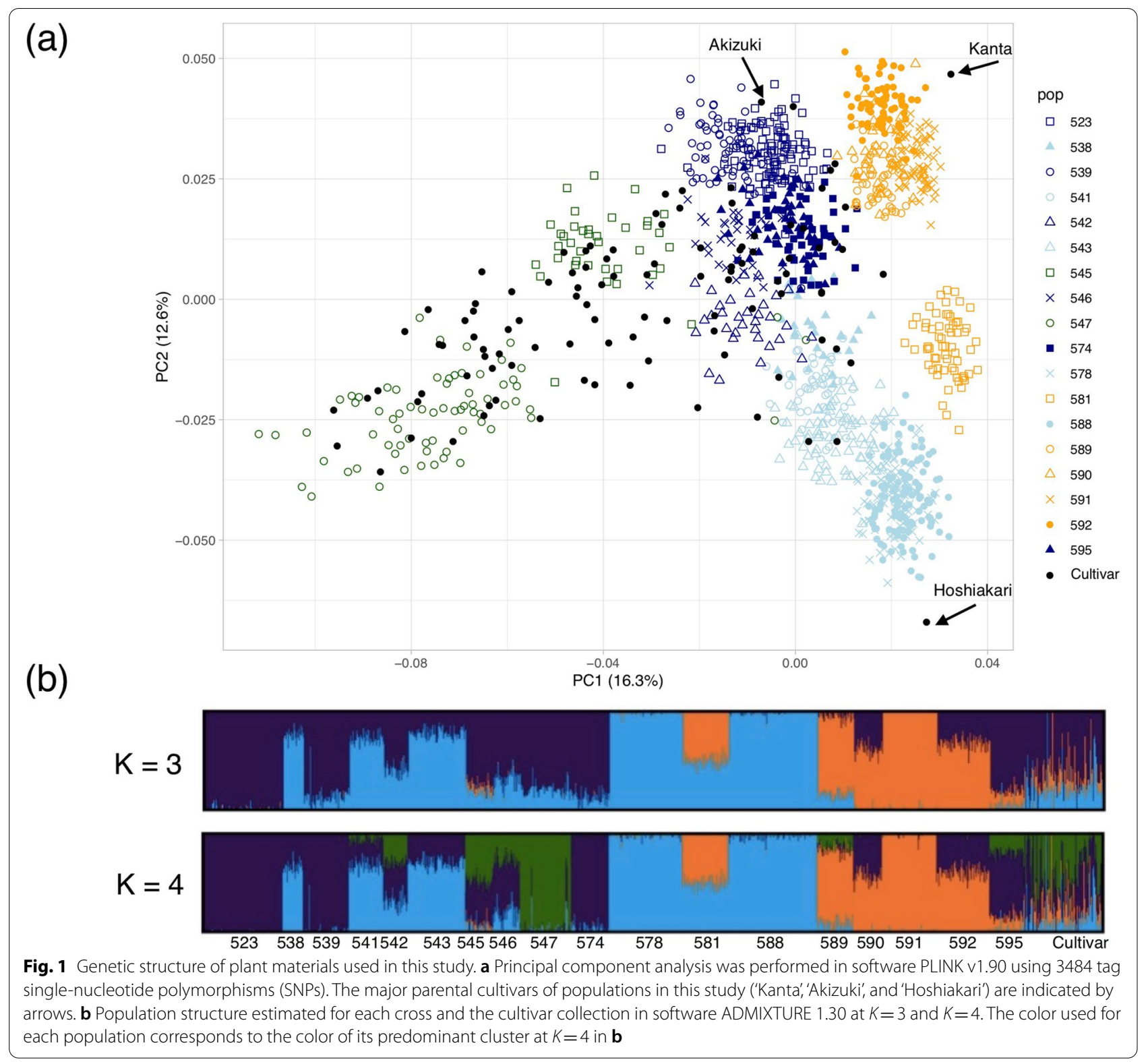

(FruH) was 2.2-9.5 lb (mean $4.8 \mathrm{lb}$ ), SSC was 10.3-18.1\% (mean $13.8 \%$ ), and acidity (Aci, measured by $\mathrm{pH}$ ), was 3.4-5.6 (mean 4.9).

Phenotypic correlation coefficients and their significances for all trait combinations were calculated (Table 2). SUC had strong negative correlations with FRU, GLC, and SOR $(r=-0.57,-0.76$, and -0.42 , respectively), and GLC had positive correlations with FRU and SOR ( $r=0.39$ and 0.36 , respectively). TSC was correlated positively with SUC, FRU, and SOR $(r=0.36$, 0.22 , and 0.29 , respectively) but was not significantly correlated with GLC. TSC had a very strong positive correlation with SSC $(r=0.92)$, indicating that the SSC of juice from mature Japanese pear was composed almost entirely of sugars.

\section{ddRAD genotyping}

A total of 1.7 billion reads were obtained from the 1218 individuals (average of $1.4 \mathrm{M}$ reads per individual). After trimming low-quality data and adapter sequences, $94.5 \%$ of the high-quality reads were successfully mapped onto the apple GDDH13 reference genome. After selecting SNP loci with VCFtools and removing individual SNP markers with $<25 \%$ missing data, we obtained 9011 SNPs. Missing data were imputed using Beagle 4.0, and 7463 
SNPs with $>0.8$ imputation accuracy were selected. To normalize the SNP density, 3484 tag SNPs were selected from the 7463 SNPs using Haploview.

\section{Linkage disequilibrium and population structure}

Linkage disequilibrium (LD) between pairs of loci was estimated using all 7463 SNPs, before selecting the tag SNPs. The $r^{2}$ values (estimates of LD) were plotted against marker distances (bp) in the apple genome GDDH13. The average $r^{2}$ values dropped below 0.2 at a marker distance of $250 \mathrm{~kb}$ and below 0.1 at $1750 \mathrm{~kb}$ (Figure S1). Population structure was estimated by principal component analysis (PCA) and Bayesian clustering analysis (Fig. 1; Table 1; Table S1). In PCA, the first principal component, which explained $16.3 \%$ of the total variation, reflected the difference between old cultivars or populations derived from relatively old cultivars (green) and populations derived from crosses among new cultivars (light blue, orange, and dark blue). The second principal component, which explained $12.5 \%$ of the total variation, reflected the genetic difference between 'Kanta' and 'Hoshiakari,' i.e., the populations derived from 'Kanta' (orange) were distributed towards positive values of the second principal component and those of 'Hoshiakari' (light blue) were distributed towards negative values.

Prior to setting the value of $K$ for Bayesian clustering analysis, the standard errors of the cross-validation error were calculated and found to plateau around $K=15$. Because the cluster diagrams for values of $K$ around $K=15$ were too complicated to provide a clear overview of the genetic relationships among the breeding populations, Bayesian clustering analyses at $K=3$ and $K=4$ are shown in Fig. 1 . We chose these values because the clusters at $K=3$ and $K=4$ roughly corresponded to the classification based on PCA. Although cultivars showed admixed genetic structure, each breeding population had a simple genetic structure. Populations derived from crosses with 'Kanta' (proportion of "orange" cluster $=1.00$ ) and other cultivars (population IDs 581, 589, 590, 591, and 592) presented the "orange" genetic cluster, those derived from crosses with 'Hoshiakari' (proportion of "light blue" $=1.00$ ) and other cultivars (IDs 538, 541, 543, 578, 581, and 588) presented the "light blue" genetic cluster, and those derived from crosses with 'Akizuki' (proportion of "dark blue" $=1.00$ ) and other cultivars (IDs 523, 538, 539 , and 546) presented the "dark blue" genetic cluster. When $K=4$, a "green" cluster was formed from the "dark blue" and the "light blue" clusters in some cultivars and populations (IDs 542, 545, 546, and 547). The average ratio of "orange":"light blue":"dark blue" in 1218 individuals was 0.20:0.38:0.42 at $K=3$, and the average ratio of "orange":"light blue":"dark blue":"green" was 0.19:0.33:0.35:0.13 at $K=4$. The new "green" cluster seemed to be derived from relatively old cultivars such as 'Okuroku' (not included in the 1218 individuals) and 'Inagi' (proportion of "green" $=0.60$ ) that are genetically somewhat different from recent cultivars.

Table 3 Molecular markers showing association with traits evaluated using a mixed linear model (MLM)

\begin{tabular}{lllllc}
\hline SNP & Trait & Chromosome & Position (bp) & -log10 (p) value & PVE (\%) \\
\hline Chr07_33139082 & SUC & 7 & 33139082 & 9.9 & 23.5 \\
Chr15_17923340 & SUC & 15 & 17923340 & 4.4 & 6.0 \\
Chr06_7938399 & FRU & 6 & 7938399 & 5.9 & 0.7 \\
Chr07_33139082 & FRU & 7 & 33139082 & 6.6 & 9.2 \\
Chr11_41197041 & FRU & 11 & 41197041 & 6.3 & 2.9 \\
Chr15_16568005 & FRU & 15 & 16568005 & 5.7 & 1.7 \\
Chr00_30710088 & GLC & 0 & 30710088 & 8.8 & 21.1 \\
Chr06_7938399 & GLC & 6 & 7938399 & 4.6 & 2.3 \\
Chr07_33139082 & GLC & 7 & 33139082 & 6.5 & 25.2 \\
Chr11_41197041 & GLC & 11 & 41197041 & 18.9 & 21.7 \\
Chr03_31587739 & HarT & 3 & 31587739 & 8.9 & 16.5 \\
Chr15_16568005 & HarT & 15 & 16568005 & 12.2 & 20.3 \\
Chr00_23086705 & ACi & 0 & 23086705 & 5.0 & 4.4 \\
Chr06_20720912 & Aci & 6 & 20720912 & 15.7 & 13.1 \\
Chr14_14932973 & Aci & 14 & 14932973 & 4.2 & 3.0 \\
Chr16_3043313 & ACi & 16 & 3043313 & 7.6 & 11.0 \\
\hline
\end{tabular}

Traits are as defined in Table 2

PVE indicates the percentage of the phenotypic variance explained by the QTL

$\mathrm{SUC}=$ sucrose content, $\mathrm{FRU}=$ fructose content, $\mathrm{GLC}=$ glucose content, $\mathrm{HarT}=$ harvest time, $\mathrm{Aci}=$ acidity 


\section{Genome-wide association study (GWAS)}

GWAS analysis was conducted for 10 fruit traits by applying two methods: one using a mixed linear model (MLM) to test the significance of association between a single SNP and each trait, and the other using a Bayesian multiple-QTL model. The latter was regarded a variational approximation version of the BayesB method [50] in which all SNPs were simultaneously fitted in the model with a variational approximation [51] (hereafter referred to as vBayesB). In the MLM method, each SNP was tested separately, so some SNPs actually linked to QTLs with minor effects were not identified as significant when there are other QTLs with large effects located near the miner effects and concealed their effects the miner QTLs. To compensate for this drawback of the MLM method, we additionally applied the vBayesB method for GWAS, in which multiple SNPs are simultaneously fitted in a statistical model for explaining phenotypic data. Because the vBayesB method is based on a multiple-SNP model, it allows detection of minor-effect QTLs even when closely linked to those with larger effects. In this method, however, the false positive rate might be slightly increased depending on the non-genetic background effects and the threshold value for significance. For covariates controlling background effects, we included the progeny effect specific to each F1 family in the model while we adopted a threshold value of 0.85 for posterior probability for SNP inclusion in the model. These settings for vBayesB were suitable for controlling the false positive rate. We considered the SNPs that were significantly detected with both methods as reliable QTLs affecting traits related to sugar contents. As shown in Figure S2, a substantial proportion of the SNPs significant with the MLM method were also assigned high posterior probabilities of being included in the model with the vBayesB method.

In the MLM-based GWAS, 16 significant SNPs were identified for five fruit traits (SUC, FRU, GLC, HarT, and Aci; Table 3). No significant SNPs were identified for SOR, TSC, FruW, FruH, or SSC. The relationships between genotype and phenotype for SNPs that explained $\geq 10 \%$ of the phenotypic variation for a trait are shown in box plots (Fig. 2). Different sugar content traits that were significantly associated with the same SNP are grouped in Fig. 2 in order to compare the relationships between marker genotype and the content of each individual sugar, possibly revealing SNPs involved in sugar conversion. Two QTLs for SUC were detected on chromosomes 7 and 15; four QTLs for FRU were detected on chromosomes 6, 7, 11, and 15; and four QTLs for GLC were detected on chromosomes $0,6,7$, and 11 . Chr07_33139082 was identified as a significant SNP for SUC, FRU, and GLC, with $23.5 \%, 9.2 \%$, and $25.2 \%$ of the phenotypic variance, respectively, explained by the SNP effects. For QTLs that had a large and significant effect on one or more individual sugar contents, the average values of all sugar content traits for each genotype are shown in Table S2. The average values of genotypes at Chr07_33139082 for AA, AC, and CC were 54.0, 39.1, and $27.4 \mathrm{mg} / \mathrm{ml}$ for SUC; $36.8,43.0$, and $42.2 \mathrm{mg} / \mathrm{ml}$ for FRU; and 10.4, 16.0, and $22.8 \mathrm{mg} / \mathrm{ml}$ for GLC, respectively (Fig. 2, Table S2), suggesting that the effect of the QTL on SUC had strong negative correlations with its effects on both FRU and GLC. On the other hand, this SNP seemed to have little effect on TSC $(131.5,130.5$, and $129.5 \mathrm{mg} /$ $\mathrm{ml}$ for the average values of $\mathrm{AA}, \mathrm{AC}$, and $\mathrm{CC}$, respectively; Table S2). For FRU, two SNPs (Chr06_7938399 and Chr11_41197041) were significant. Chr11_41197041 showed effects with negative correlation between FRU and GLC. The effect of Chr11_41197041 on GLC had the highest $-\log 10(\mathrm{p})$ value, with $21.7 \%$ of the variance explained by the SNP. The average value for GLC of each genotype at Chr11_41197041 was $10.8 \mathrm{mg} / \mathrm{ml}$ for GG, $14.3 \mathrm{mg} / \mathrm{ml}$ for GT, and $21.4 \mathrm{mg} / \mathrm{ml}$ for TT, whereas the average values for FRU were $42.8 \mathrm{mg} / \mathrm{ml}$ for GG, $40.5 \mathrm{mg} /$ $\mathrm{ml}$ for GT, and $38.1 \mathrm{mg} / \mathrm{ml}$ for TT (Fig. 2, Table S2). On the other hand, this SNP seemed to have a relatively small effect on TSC (129.4, 130.9, and $132.1 \mathrm{mg} / \mathrm{ml}$ for the average value of GG, GT, and TT, respectively; Table S2). Chr00_30710088, located on fictive chromosome 0 in the apple GDDH13 genome, was also significant for GLC, but was strongly linked to Chr11_41197041 in several populations (for example, $r^{2}=1.00$ between Chr00_30710088 and Chr11_41197041 for population 589, derived from 'Kanta' and 'Rinka'). Thus, the effect of Chr00_30710088 was excluded from further analysis and discussion. Also, Chr15_17923340, which was significant for SUC, and Chr15_16568005, which was significant for FRU, are close to each other, suggesting that these two SNPs are detecting the same QTL. For HarT, two significant SNPs were identified in the MLM-based GWAS. Chr03_31587739

(See figure on next page.)

Fig. 2 Box plots showing the association of SNP genotypes with six fruit traits. The SNPs shown explained more than 10\% of the phenotypic variance for the indicated trait(s). Box plots for different traits associated with the same SNP are enclosed by rounded rectangles. Chr15_1792340 for SUC and Chr15_16568005 for FRU, which are close to one another, are enclosed by a rounded rectangle with a dotted line. Chr04_29871378 for FRU and Chr12_4383743 for FruH were identified only in the vBayesB-based GWAS analysis (Table 4); the rest were identified in the MLM-based GWAS analysis (Table 3) or in both. The fictive chromosome 0 contains all unassigned scaffolds. For each QTL genotype, the red dot and thick horizontal line indicate the average value and median value, respectively 

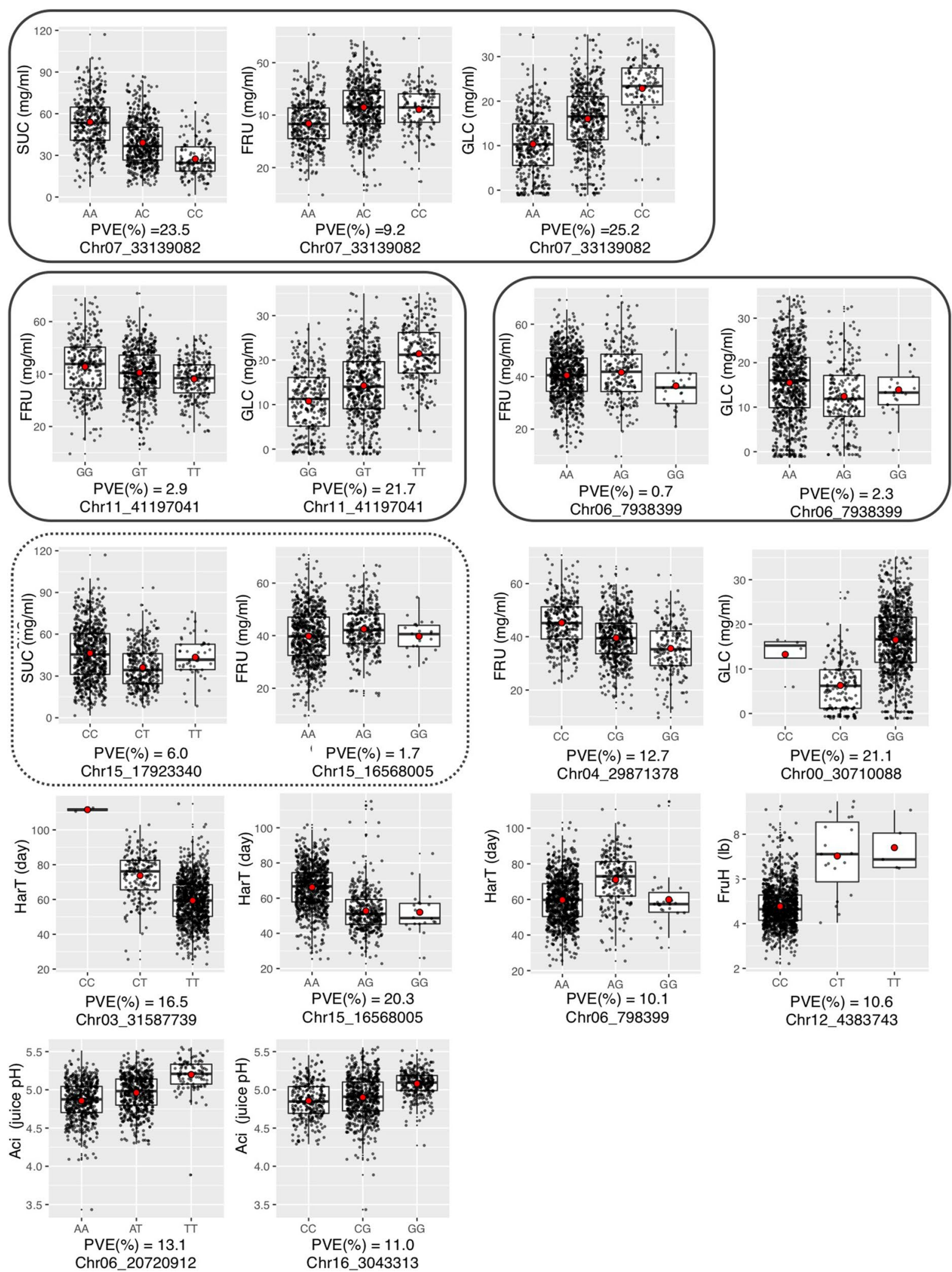

Fig. 2 (See legend on previous page.) 
Table 4 Molecular markers showing association with traits evaluated using a Bayesian multiple-QTL model (vBayesB)

\begin{tabular}{|c|c|c|c|c|c|}
\hline SNP & Trait & LG & Position (cM) & Prob & PVE (\%) \\
\hline Chr07_33139082 & SUC & 7 & 33139082 & 1.00 & 23.5 \\
\hline Chr09_18310939 & SUC & 9 & 18310940 & 0.93 & 8.8 \\
\hline Chr12_26930814 & SUC & 12 & 26930814 & 0.99 & 2.9 \\
\hline Chr04_29871378 & FRU & 4 & 29871378 & 0.97 & 12.7 \\
\hline Chr06_35256785 & FRU & 6 & 35256784 & 0.87 & 0.6 \\
\hline Chr10_8714751 & FRU & 10 & 8714751 & 0.97 & 0.3 \\
\hline Chr10_31918464 & FRU & 10 & 31918464 & 0.87 & 6.9 \\
\hline Chr11_41197041 & FRU & 11 & 41197040 & 0.99 & 2.9 \\
\hline Chr14_29691767 & FRU & 14 & 29691768 & 0.99 & 5.5 \\
\hline Chr01_27419265 & GLC & 1 & 27419264 & 0.91 & 1.2 \\
\hline Chr07_33139082 & GLC & 7 & 33139082 & 0.89 & 25.2 \\
\hline Chr10_31918464 & GLC & 10 & 31918464 & 0.87 & 8.9 \\
\hline Chr11_41197063 & GLC & 11 & 41197064 & 0.96 & 23.7 \\
\hline Chr14_28907638 & SOR & 14 & 28907638 & 1.00 & 0.4 \\
\hline Chr15_4672726 & SOR & 15 & 4672726 & 0.88 & 0.4 \\
\hline Chr02_2240743 & TSC & 2 & 2240743 & 1.00 & 2.0 \\
\hline Chr05_43292357 & TSC & 5 & 43292356 & 1.00 & 2.1 \\
\hline Chr07_9849922 & TSC & 7 & 9849922 & 0.98 & 6.5 \\
\hline Chr04_5362900 & HarT & 4 & 5362900 & 0.93 & 3.4 \\
\hline Chr05_7790403 & HarT & 5 & 7790403 & 1.00 & 0.1 \\
\hline Chr05_31445189 & HarT & 5 & 31445188 & 0.98 & 1.4 \\
\hline Chr06_7938399 & HarT & 6 & 7938399 & 0.97 & 10.1 \\
\hline Chr07_20343398 & HarT & 7 & 20343398 & 0.99 & 0.6 \\
\hline Chr13_37081601 & HarT & 13 & 37081600 & 0.97 & 1.6 \\
\hline Chr17_4322827 & HarT & 17 & 4322827 & 1.00 & 1.3 \\
\hline Chr01_30025944 & FruW & 1 & 30025944 & 0.89 & 4.9 \\
\hline Chr07_33920595 & FruW & 7 & 33920596 & 0.95 & 1.6 \\
\hline Chr09_1463613 & FruW & 9 & 1463613 & 0.89 & 2.7 \\
\hline Chr16_4765134 & FruW & 16 & 4765134 & 0.88 & 0.3 \\
\hline Chr02_29263941 & FruH & 2 & 29263940 & 0.91 & 4.6 \\
\hline Chr08_6783563 & FruH & 8 & 6783563 & 0.88 & 2.7 \\
\hline Chr12_4383743 & FruH & 12 & 4383743 & 0.87 & 10.6 \\
\hline Chr02_19653219 & SSC & 2 & 19653220 & 0.91 & 0.9 \\
\hline Chr04_30194726 & SSC & 4 & 30194726 & 1.00 & 0.2 \\
\hline Chr17_5809940 & SSC & 17 & 5809940 & 1.00 & 8.2 \\
\hline Chr01_26225120 & Aci & 1 & 26225120 & 1.00 & 1.1 \\
\hline Chr04_9535067 & Aci & 4 & 9535067 & 0.92 & 2.3 \\
\hline Chr06_20720912 & Aci & 6 & 20720912 & 0.91 & 13.1 \\
\hline Chr06_32310033 & Aci & 6 & 32310032 & 0.95 & 0.4 \\
\hline Chr09_15996669 & Aci & 9 & 15996669 & 0.90 & 0.6 \\
\hline
\end{tabular}

Traits are as defined in Table 2

Prob indicates posterior probability

PVE indicates the percentage of the phenotypic variance explained by the QTL

SUC = sucrose content, FRU = fructose content, GLC = glucose content, SOR = sorbitol content, TSC = total sugar content, HarT = harvest time, FruW = fruit weight, $\mathrm{FruH}=$ fruit hardness, $\mathrm{SSC}=$ soluble solids concentration (\%), Aci = acidity

and Chr15_16568005 showed high $-\log 10$ (p) values $(8.9$ and 12.2, respectively) and explained $16.5 \%$ and $20.3 \%$ of the phenotypic variance, respectively. The difference between the average values of the $\mathrm{CT}$ and $\mathrm{TT}$ genotypes was 14 days for Chr03_31587739, while the number of CC individuals was too low to include in the comparison. For Chr15_16568005, the average values of the AG and GG genotypes were comparable (52.7 and 52.0, 


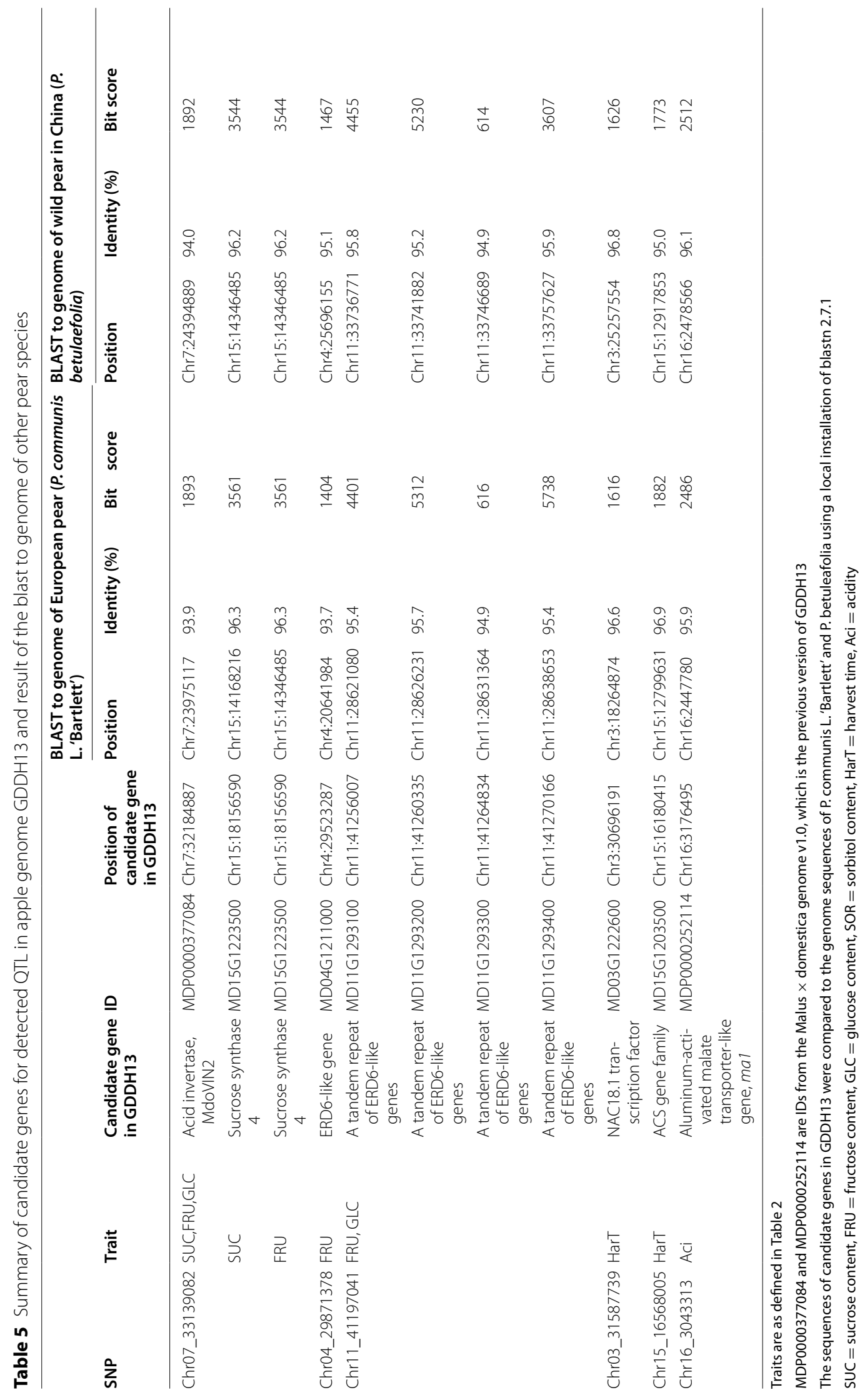


Table 6 Prediction accuracies of genomic selection for each trait based on genomic best linear unbiased prediction (GBLUP) and vBayesB-based method

\begin{tabular}{lll}
\hline Trait & GBLUP & vBayesB \\
\hline SUC & 0.72 & 0.69 \\
FRU & 0.67 & 0.60 \\
GLC & 0.75 & 0.75 \\
SOR & 0.63 & 0.58 \\
TSC & 0.55 & 0.39 \\
HarT & 0.74 & 0.65 \\
FruW & 0.56 & 0.46 \\
FruH & 0.56 & 0.41 \\
SSC & 0.57 & 0.45 \\
Aci & 0.64 & 0.66 \\
\hline
\end{tabular}

Values indicate Pearson's correlation coefficient $(r)$ between predicted genotypic values and phenotypic values

respectively), but that of AA was 66.2. Out of the four significant SNPs associated with Aci, Chr06_20720912 and Chr16_3043313 showed relatively high percentages of variance explained (13.1\% and $11.0 \%$, respectively). The average values for Aci at Chr06_20720912 were 4.85 for AA, 4.96 for AT, and 5.20 for TT (Fig. 2), and those at Chr16_3043313 were 4.85 for CC, 4.90 for CG, and 5.08 for GG (Fig. 2).

In the vBayesB-based GWAS, 40 SNPs showed posterior probabilities exceeding 0.85 for all 10 fruit traits (Table 4, Table S2). Out of those 40 SNPs, 4 were also significant in the MLM-based GWAS: Chr07_33139082 for SUC and GLC, Chr11_41197041 for FRU, and Chr06_20720912 for Aci. Chr11_41197063 (for GLC) is close to Chr11_41197041, suggesting that they are associated with the same gene. Although the posterior probability values were high, the percentages of phenotypic variance explained by the SNPs were not large for most of the detected SNPs. Out of the 40 SNPs, only 7 had percentages of phenotypic variance explained of $\geq 10 \%$, including the four that were also significant in MLM-based GWAS (considering Chr11_41197063 to be the same locus as Chr11_41197041). In addition to those four, Chr04_29871378 explained $12.7 \%$ of the phenotypic variance for FRU, and the average value of each genotype was $45.3 \mathrm{mg} / \mathrm{ml}$ for CC, $39.6 \mathrm{mg} / \mathrm{ml}$ for $\mathrm{CG}$, and $35.6 \mathrm{mg} / \mathrm{ml}$ for GG (Fig. 2). These values were positively correlated with those for TSC (134.2 mg/ $\mathrm{ml}$ for CC, $129.8 \mathrm{mg} / \mathrm{ml}$ for CG, and $127.3 \mathrm{mg} / \mathrm{ml}$ for GG; Table S2), though this SNP was not detected in the vBayesB-based GWAS for TSC. Chr06_7938399 (for HarT) and Chr12_4383743 (for FruH; see Fig. 2) each accounted for $10 \%$ or more of the phenotypic variance for the indicated trait, but these SNP had minor genotype frequency $(<0.05)$ and did not segregate in most of the populations.
To illustrate the effects of the 56 SNPs from the two GWAS analyses in each population, the percentages of phenotypic variance explained by SNPs in each population were calculated and displayed in a heatmap (Figure S3). While the SNPs identified in MLM-based GWAS tended to show effects across the 1218 individuals, some SNPs identified in vBayesB-based GWAS showed effects specific to a population. For example, Chr04_5362900 (for HarT) and Chr12_4383743 (for FruH) explained high percentages of variance only in the cultivar collection.

Based on the QTLs and genes previously identified in apple, we searched for candidate genes for fruit traits in the GDDH13 genome (Table 5). For the QTLs associated with individual sugar contents, we found an acid invertase gene, a sucrose synthase gene, and ERD6-like genes as candidates on apple chromosomes $4,7,11$, and 15. For the QTLs for fruit harvesting day, a gene for the NAC18.1 transcription factor was found on chromosome 3 , and a member of ACS gene family was found on chromosome 15. An aluminum-activated malate transporterlike gene, $\mathrm{ma1}$, was also detected on chromosome 16 as a candidate for acidity. When we searched for the locations of these candidate genes in European pears and wild pear in China by using BLAST + , all of the candidate genes were detected in similar regions of both genomes.

\section{Genomic selection}

We attempted to predict the breeding values of individuals with genomic best linear unbiased prediction (GBLUP), in which a polygenic effect included in MLMbased GWAS was regarded as the breeding value of an individual, and with the vBayesB-based method, which was also used in GWAS. We used each F1 family as a tested population and the remaining families and cultivars as the training population, where a prediction model was constructed with both observed phenotypes and 
SNP genotypes of a training population and the breeding values of each tested population were predicted from SNP genotypes with the constructed model. We calculated correlation coefficients between predicted breeding values and observed phenotypes over all individuals in F1 families (Table 6). The prediction accuracies in GBLUP were higher than those in the vBayesB-based method for SUC, FRU, SOR, TSC, HarT, FruW, FruH, and SSC, whereas the prediction accuracies in GBLUP for GLC and Aci were the same as or slightly lower than those in the vBayesB-based method. GLC showed the highest values of prediction accuracy in both GBLUP and the vBayesB-based method (0.75). TSC, FruW, FruH, and SSC showed lower values of prediction accuracy, especially with the vBayesB-based method (0.39-0.46).

\section{Discussion}

Several phenotypic correlations among individual sugars were identified in 1218 individuals and cultivars. SUC had strong negative correlations with both FRU and GLC ( $r=-0.57$ and -0.76 , respectively), and FRU had a positive correlation with GLC $(r=0.39)$. This result was quite similar to those in previous studies of QTLs for individual sugar traits [9] and genetic differences in individual sugars among leading or promising cultivars [52]. In addition, SOR had a negative correlation with SUC in this study $(r=-0.42)$. These results suggested that sugar conversion from sucrose to hexose or from sorbitol to sucrose had a strong influence on sugar composition in mature fruit. TSC had quite a strong correlation with SSC, suggesting that it would be possible to use SSC for the first screening of individuals: it is much easier to measure SSC (the amount of sugar in fruit) than TSC, which requires high-performance liquid chromatography. In a previous study, the broad-sense heritability of TSC was only 0.33 , whereas those of SUC, FRU, GLC, and SOR were $0.64,0.69,0.71$, and 0.76 , respectively [52]. Segregating TSC into individual sugars would be an effective way to identify the genes associated with sugar accumulation and conversion in mature fruit, because the low heritability of TSC would make genetic analyses of that trait difficult. HarT had positive correlations with FruW, TSC, and SSC, as reported in previous studies $[10,53]$. This suggests that it might be difficult to develop earlyripening cultivars with large fruit size and high sugar content. On the other hand, FruH and Aci did not show clear correlations with other fruit traits, suggesting that they are independent and controlled by different genes.

In previous studies, LD values of Japanese pear had been calculated based on genetic linkage maps (centimorgan distance) $[8,10]$. In this study, we calculated LD based on the apple physical map, enabling us to compare the LD blocks with those in apple. The average $r^{2}$ values dropped below 0.2 at $250 \mathrm{~kb}$. Our material had a smaller LD block size than the apples studied by Moriya et al. [54] and Kumar et al. [55], but a larger LD block size than in the study of Leforestier et al. [56]. We expected that the LD in Japanese pear would be high because genetic bottlenecks and breeding history would have increased the extent of LD by eliminating recombinant lineages [8]. Nevertheless, the LD block size was smaller than in several apple genetic studies, suggesting that apple experienced a strong bottleneck similar to that presumed to have occurred during pear breeding and domestication. In the present study, the cultivar collection had admixed structure in Bayesian structure analyses and showed broad distribution in PCA compared to individual populations, whereas each population had smaller genetic diversity than the cultivar collection. In the Bayesian structure analysis at $K=4$, the populations were roughly divided into four groups: those derived from crosses between 'Kanta' and other cultivars ("orange"), those derived from crosses between 'Hoshiakari' and other cultivars ("light blue"), those derived from crosses between 'Akizuki' and other cultivars ("dark blue"), and cultivars and populations relatively distant from those three cultivars ("green"). 'Kanta' has high sweetness, with high TSC and FRU [52], 'Hoshiakari' has a scab resistance gene inherited from local cultivar 'Kinchaku' [57], and 'Akizuki' has a good fruit texture with excellent fruit shape. Since each of these cultivars has specific desirable characteristics, they have been used as parents in Japanese pear breeding programs. In essence, the populations used in this study were based on only 13 founder cultivars [57]. Because of the loss of genetic diversity among modern cultivars [36], the genetic diversity of the populations used in this study was not very large. The genetic structure identified in this study reflects the small genetic differences between the parental cultivars used to create the populations, which basically represent the same gene pool, rather than ancestral populations derived from different gene pools.

In this study, we identified several candidate genes based on the genome sequence of GDDH13. All of these candidate genes were successfully mapped to the wholegenome sequences of European pear and wild pear in China, suggesting that those genes are conserved in the Pyrinae (Table 5). We identified a QTL associated with conversion of SUC to FRU and GLC on chromosome 7. A previous study also identified a QTL associated with conversion of SUC to FRU and GLC in this region (chromosome 7) and on chromosome 1, and further suggested that acid invertase genes PPAIV1 and PPAIV3 were the candidate genes [9]. In apple, a similar QTL for conversion of individual sugars was also identified on 
chromosome 1 in both mature fruit and fruit after storage [28]. Thus, it is possible that the AIV gene family plays an important role in determining individual sugar contents in mature fruit of Rosaceae fruit species. A candidate gene for QTLs that were significant for SUC and FRU on chromosome 15 (Chr15_17923340 and Chr15_16568005) might be sucrose synthase 4 (MD15G1223500, Chr15:18,156,590-18,162,358). Another possibility is sucrose phosphate synthase 1F (MD15G1164900, Chr15:12,407,116-12,413,753), though it is located at a distance of about $500 \mathrm{~kb}$ from the detected SNP. Because sucrose synthase catalyzes the reversible conversion of sucrose and UDP to UDP-glucose, and sucrose phosphate synthase catalyzes the synthesis of sucrose from sucrose 6-phosphate, it is reasonable that these genes are related to the QTLs on chromosome 15. Chr11_41197041 was identified as a significant SNP for both FRU and GLC, showing a strong effect in several populations (Table S2). Because a negative correlation between FRU and GLC was observed for this SNP, it would potentially enable us to select genotypes with high FRU and low GLC, thus improving fruit sweetness. The QTL identified at Chr04_29871378 for FRU explained 12.7\% of the variance among 1218 individuals and showed relatively high percentages of variance in several populations (more than $10 \%$ of the variance explained in nine populations; Figure S3). The effect of the QTL was limited to FRU; other individual sugars seemed to be unaffected, suggesting that an increase in FRU is directly associated with an increase in TSC (Table S2).

Interestingly, early responsive to dehydration (ERD6)-like gene, encoding sugar transporters, are also located near two of the SNPs (Chr11 41197041 and Chr04_29871378). A single ERD6-like gene was detected near Chr04_29871378 (MD04G1211000; Chr4:29,523,287-29,525,923), whereas a tandem repeat of ERD6-like genes (MD11G1293100, MD11G1293200, MD11G1293300, MD11G1293400; Chr11:41,256,00741,275,197) was detected near Chr11_41197041. Arabidopsis ERD6 was found to be sugar transporters in bacteria, yeasts, other plants, and mammals [58]. This transporter was reported to be localized to the tonoplast, acting as an $\mathrm{H}+/$ glucose symporter to facilitate the export of glucose from the vacuole to the cytosol and regulating cellular glucose in response to various stresses [59]. Tandem duplications of the ERD6-like family genes were found to be conserved in citrus, grape, apple, and Chinese jujube [60]. The order of the tandem repeat of ERD6-like genes detected in this study is also conserved in the genomes of European pear and wild pear in China (Table 5). Zhang et al. [61] suggested that duplication of sugar transporter genes plays crucial roles in sugar accumulation. Because Chr11_41197041 was the SNP with the largest effect on GLC, explaining $21.7 \%$ of the phenotypic variance, it is reasonable that transport of glucose from the vacuole to the cytosol by this transporter contributed to the observed changes in the individual sugar contents in mature fruit. The vacuole of mature fruit can occupy more than $90 \%$ of the cell volume [62], storing sugars and other compounds. Export of glucose instead of $\mathrm{H}^{+}$by the product of this gene may produce an electrochemical gradient across the vacuolar membrane and bring other sugars into the vacuole.

Several QTLs for HarT and Aci similar to those identified here were identified in previous studies of apple. The NAC18.1 transcription factor (MD03G1222600; Chr3:30,696,191-30,698,216) is a promising functional candidate for fruit ripening [63] and is close to Chr03_31587739 (for HarT) in this study. A NAC-family genes were also associated with maturity date and slow ripening in peach $[64,65]$. One member of the ACS gene family (MD15G1203500) is located at Chr15:16,180,415, which is close to Chr15_16568005 (for HarT) in this study. This QTL and the function of the ACS gene have already been analyzed in several studies and found to affect fruit harvesting day, storage ability, and fruit drop [11, 34-36]. The early-harvesting genotype is correlated with increased fruit drop and short storage ability, suggesting that pear breeders need to consider carefully whether they use this marker for MAS, depending on their breeding objectives [38]. In apple, an aluminum-activated malate transporter-like gene (ma1, MDP0000252114) was identified as the candidate for a gene associated with low fruit acidity [66]. In the apple GDDH13 genome, ma1 is located at Chr16:3,176,495-3,179,279, which is close to Chr16_3043313 (for Aci) in this study. In apple, two major loci, Ma3 on chromosome 8 and Ma1 on chromosome 16, play an important role in fruit acidity [67]. On the other hand, another major locus for Aci in this study was identified on chromosome 6: this locus showed quite a large effect and explained more than $30 \%$ of the phenotypic variance in seven populations (Chr06_20720912 in Figure S3).

In this study, we focused on detecting QTLs and inferring putative candidate genes for those QTLs, whereas Minamikawa et al. [10] focused on the accuracy of various methods for genomic prediction rather than on inferring candidate genes. We estimated prediction accuracy for GS using GBLUP and a vBayesBbased method for GWAS analyses. Most traits showed higher prediction accuracies with GBLUP than with the vBayesB-based method; on the other hand, the prediction accuracy for Aci was slightly higher when the vBayesB-based method was used. It is natural that the 
best GS model varies depending on the trait because the distribution of the trait values vary depending on heritability, allele frequencies, and the population structure of the materials. It will be important to choose the appropriate model for each trait. The prediction accuracies for SUC, FRU, and GLU with GBLUP were relatively high $(0.67-0.75)$, suggesting that it would be possible to select individuals that have high-sweetness fruit (high SUC and FRU, and low GLU). On the other hand, the prediction accuracy for TSC, which is the most important trait determining fruit quality, is not high enough for use in GS ( 0.55 in GBLUP) There is room to improve the prediction accuracy by constructing the genome sequence of Japanese pear and using it as a reference genome, thus increasing the number of SNPs and improving map precision. The accuracy of genomic prediction can also be improved further when full-sib data for the target family are available [10]. It would be a good idea to apply GS to the populations used in this study, i.e., those derived from the crosses between 'Hoshiakari', 'Kanta', 'Akizuki', and other cultivars. The practical way to introduce genomic selection in breeding programs is to predict the genetic values of each trait of seedings using next-generation sequencing and the best fitting model, and to undercut the seedlings that have lower predicted values before planting in breeding fields. The selecting intensity can be flexibly determined by breeders based on the objective of breeding programs, the number of the seedlings and size of the breeding fields.

\section{Conclusions}

In this study, we collected phenotypic data for fruit traits and conducted GWAS and GS in Japanese pear. Several important QTLs for fruit traits were identified, and genes associated with sugar accumulation were predicted by GWAS using a large number of individuals. The SNP located closest to PPAIV3 on chromosome 7 and a newly identified SNP at chromosome 11 had large effects on individual sugar contents. The SNP on chromosome 4 that was associated with FRU would be useful for increasing the contents of FRU and TSC without decreasing the contents of other individual sugars. The candidate genes of QTLs identified in this study are conserved in the genomes of several Pyrinae species. Further studies including expression analysis of those genes and developing gene-specific markers would contribute to clarifying the mechanisms of sugar accumulation and validating the candidate genes for fruit traits. Fruit traits are complex and controlled by multiple factors, so it is important to accumulate relevant genetic information. The traits evaluated in this study covered the principal fruit traits in pear breeding programs, so the results obtained illustrate the feasibility of GS for fruit traits in pear.

\section{Methods \\ Plant materials}

A cultivar collection including 106 cultivars and 17 breeding populations (consisting of $1112 \mathrm{~F} 1$ individuals) were used in this study (Table 1, Table S1). The cultivars were preserved at the NARO (National Agriculture and Food Organization) Genebank (www.gene.affrc.go.jp) and the breeding populations were developed at Institute of Fruit Tree and Tea Science, NARO. The population ID numbers are indicated in Table 1 , and each family contained 28 to 121 individuals. Among those materials, 74 cultivars and 498 individuals were the same as those used in Minamikawa et al. [10]. The breeding populations originated from local cultivar 'Nijisseiki' and are the product of about five to seven generations of crossing in the NIFTS pear breeding program. The cultivars and F1 individuals were grown with cultural techniques used in commercial production in Japan [68]. The trees were trained on horizontal trellises, pruned annually in winter, and treated for pests and diseases. Fruits were thinned to one fruit per three fruit clusters in mid-May and harvested during late July to early November according to a color chart that indicates the optimum color for picking Japanese pear [69].

Ten fruit traits were evaluated: SUC, FRU, GLC, SOR, TSC, HarT, FruW, FruH, SSC, and Aci. The contents of each sugar (SUC, FRU, GLC, SOR) were analyzed in fruit from each individual. To do this, the juice from two fruits per sampling date was extracted and the samples were combined. Sampling was performed on two days in each of the years that the individual or cultivar was analyzed. The analysis of sugar components was based on the method described by Nishio et al. [9]. TSC was calculated by summing the contents of the four sugars. The harvest date for each fruit was expressed as the number of days after June 30 (i.e., July $1=$ day 1 ), and the average value of harvest date for each fruit was used as its phenotypic score. Fruit weight (g) per fruit (FruW) was measured on a digital scale on each harvest date. Fruit hardness (FruH) was measured by the Magness-Taylor pressure test (lb). Total SSC was determined with a digital refractometer (DBX-55; Atago, Tokyo, Japan) by adding a few drops of juice onto the lens of the measuring device, and the results were recorded as SSC (\%). Juice $\mathrm{pH}$ was determined with a pH meter (IQ240; Scientific Instruments, San Diego, CA, USA) to estimate acidity (Aci).

Phenotypic data were collected from 2014 to 2018. The majority of individuals and cultivars were evaluated for more than one year. The average phenotypic values over 
five years (2014-2018) were generated by the allEffects function from the package "effects". Phenotypic correlation coefficients and their significance were calculated for all trait combinations using $\mathrm{R}$ (4.0.0) (R Development Core Team).

\section{SNP genotyping}

Genomic DNA was extracted from young leaves with a DNeasy Plant Mini Kit (Qiagen, Hilden, Germany) according to the manufacturer's instructions. ddRADSeq libraries were constructed as described in Shirasawa et al. [49]. A total of $200 \mathrm{ng}$ of genomic DNA for each individual was double digested with PstI and MspI (FastDigest restriction enzymes; Thermo Fisher Scientific, Waltham, MA, USA), ligated to adapters using the LigaFast Rapid DNA Ligation System (Promega, Madison, WI, USA), and purified using Agencourt AMPure XP (Beckman Coulter, Brea, CA, USA) to eliminate short $(<300 \mathrm{bp})$ DNA fragments. Purified DNA was diluted with $\mathrm{H}_{2} \mathrm{O}$ and amplified by 20 cycles of PCR with indexed primers. Amplicons were pooled and separated on a BluePippin 1.5\% agarose cassette (Sage Science, Beverly, MA, USA), and fragments of 300-900 bp were purified using a Mini Elute Kit (Qiagen). The library was then sequenced using a HiSeq 4000 (Illumina, Inc., San Diego, CA, USA).

SNPs and indels were identified according to Acquadro et al. [70]. Illumina reads were de-multiplexed on the basis of the Illumina TruSeq index. Raw reads were analyzed with Scythe (https://github.com/vsbuffalo/scythe) for filtering out contaminant substrings and Sickle (https://github.com/najoshi/sickle), which removes reads with poor-quality ends $(\mathrm{Q}<30)$. Alignment to the reference apple genome (GDDH13 v1.1; https://www.rosac eae.org/species/malus/malus_x_domestica/genome_ GDDH13_v1.1) was carried out using BWA aligner [71] (e.g., mem command) with default parameters and avoiding multiple-mapping reads. SNP and indel mining was conducted by adopting a Samtools-based pipeline [72]. SNPs and indels (hereafter called SNPs) detected from the alignments were filtered with VCFtools (version 0.1.13; parameters: -minQ 20 -minDP 10 -maf 0.01 maf 0.99 -min-allele 2 -max-allele 2). Individual SNP markers with $>25 \%$ missing data were removed from further analysis, and the remaining missing values were phased and imputed using Beagle v4.1 [73]. Only the SNPs with an imputation accuracy $>0.8$ were adopted. The tagger software implemented in Haploview (http:// www.broad.mit.edu/mpg/haploview) was used to select tag SNPs using its pairwise function, with a minimum $r^{2}$ of 0.8 .

The draft genomes of Chinese pear (P. bretschneideri), wild pear in China (P. betulaefolia), and European pear
(P. communis) are all currently available [42-44]. However, the draft genome of Chinese pear was constructed in 2013 and we found that it contains many assembly errors. On the other hand, the genome sequences of European pear and wild pear in China are presumed to have a high level of accuracy, but they are completely different species from Pyrus pyrifolia. There is also less information on genes and transcripts and fewer relevant genetic studies in comparison with those using apple reference genomes. Because the apple genome GDDH13 is the most frequently used reference genome in the Rosaceae and it has precise order and rich information on transcripts, QTLs, and LDs, we used GDDH13 as the reference genome in this study.

\section{LD and population structure}

LD was estimated using data after the imputation. Based on the apple GDDH13 genome, the detected SNPs were aligned according to their positions. LD between pairs of SNPs was calculated using R (4.0.0) (R Development Core Team). The SNPs mapped on the fictive chromosome 0 , which contains all unassigned scaffolds, were removed to calculate the LDs. Average LD values $\left(r^{2}\right)$ were plotted against physical distances in increments of $10 \mathrm{~kb}$.

Population structure was estimated for each cross and for the cultivar collection in ADMIXTURE 1.30 [74] using the 3484 tag SNPs. The software PLINK v1.90 [75] was used to generate an input file from a vcf file. The analysis was run five times for each value of $K$ (number of inferred ancestral populations) from 1 to 20 to estimate the cross-validation values. The cross-validation value gradually decreased as the value of $K$ increased. The CLUMPAK online tool [76] was applied to graphically display the results produced by ADMIXTURE at $K=3$ and $K=4$. PCA was performed in PLINK v1.90. Prior to PCA, an input file was created by conducting linkage pruning using the -indep-pairwise option in PLINK (plink -file data -indep-pairwise 5010 0.1). PCA was performed using the -pca option in PLINK. The plot was drawn with the R package "ggplot" [77].

\section{Statistical methods in GWAS}

For the MLM-based GWAS, the effect of a SNP and a polygenic effect affected by the genetic background of an individual were included in the model as a fixed effect and a random effect, respectively. The covariance matrix of polygenic effects between individuals was established with a kinship matrix calculated from SNP genotypes. The effects of population structure were also included as fixed effects using the first three principal components obtained from the SNP genotype data. We used the $\mathrm{R}$ package rrBLUP ver. 4.3 [78] for this MLM-based GWAS 
and evaluated the effects of the significant SNPs on the basis of the mean phenotypic value for each genotype of the SNP.

For the vBayesB-based GWAS, a Bayesian multiple-QTL model was applied in which all SNPs were simultaneously fitted in the model assuming a specific probability for each SNP included in the model, called SNP weight, and a specific variance for each SNP effect as well as the progeny effect specific to each F1 family. The progeny effect specific to each F1 family was also included in the model to control for the difference in genetic background between F1 families. The model was fitted with a variational approximation method proposed by Hayashi and Iwata [51] using a custom program written in Fortran. This Bayesian procedure was regarded as a variational approximation of BayesB [51] and was previously applied to GWAS in chestnut [79], in which the adopted estimation methods were described (we refer the reader to this paper for computational details). The settings of hyperparameter values of the prior distributions were conducted following this paper [79].

To validate the effect of the detected SNPs, the average values of each genotype for the SNPs and the distribution of the phenotypic values in the SNP genotypes were plotted. These plots were drawn with the R package "ggplot" [77]. The phenotypic variance explained by each SNP was calculated according to Nishio et al. [36]. The percentage of the phenotypic variance explained by each SNP was calculated by dividing the variance of the SNP by the total phenotypic variance.

To infer the candidate genes of the detected QTLs, we thoroughly checked the QTLs and genes identified in previous apple genetic studies [28, 38-40, 42, 55-57, $63,66,67]$. If genes related to sugar synthesis, sugar conversion, or sugar transport were located within $200 \mathrm{~kb}$ of a detected QTL, they were listed in Table 5. Similarly, if previously identified genes for other fruit traits were located within $200 \mathrm{~kb}$ of a detected QTL, they were added to Table 5. BLAST $+[80]$ was used to check whether these genes were located at similar positions in the whole-genome sequences of European pear [42] and wild pear in China [44].

\section{Accuracies of predicted genomic breeding values in GS}

The two methods used in GWAS, MLM-based and vBayesB-based, were also used for prediction of breeding values based on SNP genotypes. In the MLMbased method, a polygenic effect was regarded as a breeding value that was predicted from SNP genotypes through a kinship matrix. This MLM-based method was referred to as GBLUP when applied to GS. In this study, an intercept but no fixed effects were included in MLM for GS. In the vBayesB method, we obtained estimates of a SNP effect and a SNP weight for each SNP, regarded as an approximate posterior probability of each SNP included in the model; accordingly, the predicted breeding value of an individual was calculated as the sum of the estimated SNP effects multiplied by the SNP weights over all SNPs.

Generally, in practical breeding programs, new elite cultivars and selections have been used as parents to create breeding populations and to select genotypes superior to those of the established leading cultivars. Thus, GS would be applied in breeding populations obtained by crossing between new combinations of parental genotypes. To evaluate the accuracy of genomic prediction for practical pear breeding programs, we conducted a cross-validation in the following way. Each F1 family was regarded as a tested family, and the set of cultivars and F1 families with the tested family excluded was regarded as a training population. A prediction model was constructed using data for both phenotypes and SNP genotypes of a training population, and breeding values of individuals of a tested F1 family were predicted with a model using only their SNP genotypes. This process was repeated until all F1 families were selected just once as a tested population and the prediction accuracy was evaluated with the Pearson's correlation coefficient $(r)$ between observed phenotypic values and predicted genotypic values. When the estimated $r$ was less than 0 , it was regarded as 0 . The prediction accuracy for each tested family based on the training population (which excluded the target family) was calculated and averaged to understand the accuracies of predicted genomic breeding values for the breeding populations used in this study.

\section{Availability of data and materials}

The datasets supporting the conclusions of this article are included within the article and its supplementary information files. Sequence reads are available from the Sequence Read Archive (DRA) of DNA Data Bank of Japan (DDBJ) under the accession number of DRA011324 (https://ddbj.nig.ac.jp/DRASearch/submission?acc $=$ DRA011324).

\footnotetext{
Abbreviations

Aci: Acidity; ACS: 1-Aminocyclopropane-1-carboxylate synthase; FRU: Fructose content; FruH: Fruit hardness; FruW: Fruit weight; GBLUP: Genomic best linear unbiased prediction; GDDH: 'Golden Delicious' doubled haploid; GLC: Glucose content; GS: Genomic selection; GWAS: Genome-wide association study; HarT: Fruit harvest time; MLM: Mixed linear model; NIFTS: Institute of Fruit Tree and Tea Science, NARO; PCA: Principal component analysis; SNP: Single-nucleotide polymorphism; SOR: Sorbitol content; SSC: Soluble solids concentration; SSR: Simple sequence repeat; SUC: Sucrose content; TSC: Total sugar content.
} 


\section{Supplementary Information}

The online version contains supplementary material available at https://doi. org/10.1186/s12870-021-03130-2.

\begin{abstract}
Additional file 1: Figure S1 Plots of average linkage disequilibrium values ( $\mathrm{r} 2$ ) against physical distances in increments of $10 \mathrm{~kb}$. Gray curves show local polynomial fits obtained using kernel smoothing regression. Dotted lines indicate the genetic distance at which $\mathrm{r} 2$ fell below 0.2. Figure S2 Summary of the results of genome-wide association studies using two different models. a Manhattan plots of $-\log 10(p)$ values based on a MLM for 10 fruit traits. Red lines indicate a false discovery rate of 0.05 . Chromosome location information is based on GDDH13 Version 1.1. The fictive chromosome 0 contains all unassigned scaffolds. b Posterior probability of having a QTL based on vBayesB, estimated for 10 fruit traits. Figure S3 Heatmap showing the percentage of the phenotypic variance explained by each QTL in each population. The percentages for the cultivar collection (designated as "Cultivar") and for all 1218 accessions including the populations and cultivars (designated as "ALL") are shown on the right. The prefix rrBLUP indicates the SNPS identified in the MLM-based GWAS analysis; BayesB indicates those identified in the vBayesB-based GWAS. A blank indicates that the population showed no segregation for the SNPs. The intensity of the red color corresponds to the percentage of phenotypic variance explained
\end{abstract}

Additional file 2: Table S1 The cultivars and individuals used in this study and the performance for fruit traits.

Additional file 3: Table S2. Average values of individual and total sugar contents for each genotype of three SNPs having a large and significant effect on at least one trait.

\section{Acknowledgements}

We are deeply indebted to all the people involved in the Japanese pear breeding program at the Institute of Fruit Tree and Tea Science, NARO, Japan.

\section{Authors' contributions}

SN participated in the design of the experiments, genotyped SNPS, interpreted the data, and wrote the paper. TH, KS, and SM contributed reagents and analysis tools. TS, NT, ST, and YT prepared the plant materials. Al interpreted the data and contributed funding. All the authors read and approved the manuscript.

\section{Funding}

This work was supported by the Cabinet Office, Government of Japan, Crossministerial Strategic Innovation Promotion Program (SIP), "Technologies for Smart Bio-industry and Agriculture" (funding agency: Bio-oriented Technology Research Advancement Institution, NARO). The funders had no role in study design, data collection and analysis, decision to publish, or preparation of the manuscript.

\section{Declarations}

\section{Ethics approval and consent to participate}

Not applicable.

\section{Consent for publication}

Not applicable.

\section{Competing interests}

The authors declare no competing interests.

\section{Author details}

'Institute of Fruit Tree and Tea Science, NARO (NIFTS), 2-1 Fujimoto, Tsukuba, Ibaraki 305-8605, Japan. ${ }^{2}$ Research Center for Agricultural Information Technology, NARO, 3-1-1 Kannondai, Tsukuba, Ibaraki 305-8666, Japan. ${ }^{3}$ Kazusa DNA Research Institute, 2-6-7 Kazusa-Kamatari, Kisarazu, Chiba 292-0818, Japan. ${ }^{4}$ Institute of Fruit Tree and Tea Science, NARO, Morioka, I wate 020-0123, Japan. ${ }^{5}$ Graduate School of Life and Environmental Sciences, Kyoto Prefectural University, 74 Kitainayazuma, Seika, Kyoto 619-0244, Japan.
Received: 28 January 2021 Accepted: 9 July 2021

Published online: 16 August 2021

\section{References}

1. Silva GJ, Souza TM, Barbieri RL, Oliveira ACD. Origin, domestication, and dispersing of pear (Pyrus spp.). Adv Agr. 2014;2014:e541097.

2. Kikuchi A. Speciation and taxonomy of Chinese pears. Collected Records of Hort Res Kyoto Univ. 1946;3:1-8.

3. Gu C, Sponberg SA. Pyrus: In: Wu Zy, Raven PH, Honf DY, editors. Flora of China. Vol. 9. Beijing: Science Press; 2003. p.173-19.

4. Yü TT, Ku TC. Pyrus. In: Yü TT, editor. Flora Reipublicae Popularis Sinicae, vol. 36. Beijing: Science Press; 1974. p. 354-72.

5. Iketani H, Yamamoto T, Katayama H, Uematsu C, Mase N, Sato Y. Introgression between native and prehistorically naturalized (archaeophytic) wild pear (Pyrus spp.) populations in Northern Tohoku, Northeast Japan. Conserv Genet. 2010;11:115-26.

6. Jiang ZW, Tang FY, Huang HW, Hu HJ, Chen QL. Assessment of genetic diversity of Chinese sand pear landraces (Pyrus pyrifolia Nakai) using simple sequence repeat markers. HortScience. 2009;44:619-26.

7. Nishio S, Takada N, Saito T, Yamamoto T, Iketani H. Estimation of loss of genetic diversity in modern Japanese cultivars by comparison of diverse genetic resources in Asian pear (Pyrus spp.). BMC Genet. 2016;17:81

8. Iwata H, Hayashi T, Terakami S, Takada N, Sawamura Y, Yamamoto T. Potential assessment of genome-wide association study and genomic selection in Japanese pear Pyrus pyrifolia. Breeding Sci. 2013;63:125-40.

9. Nishio S, Saito T, Terakami S, Takada N, Kato H, Itai A, et al. Identification of QTLs associated with conversion of sucrose to hexose in mature fruit of Japanese pear. Plant Mol Biol Rptr. 2018;36:643-52.

10. Minamikawa MF, Takada N, Terakami S, Saito T, Onogi A, Kajiya-Kanegae $\mathrm{H}$, et al. Genome-wide association study and genomic prediction using parental and breeding populations of Japanese pear (Pyrus pyrifolia Nakai). Sci Reports. 2018:8:11994.

11. Yamamoto T, Terakami S, Takada N, Nishio S, Onoue N, Nishitani C, et al. Identification of QTLs controlling harvest time and fruit skin color in Japanese pear (Pyrus pyrifolia Nakai). Breeding Sci. 2014;64:351-61.

12. Yamaki S. Metabolism and accumulation of sugars translocated to fruit and their regulation. J Jpn Soc Hort Sci. 2010;79:1-15.

13. Kliewer WM. Sugars and organic acids of Vitis vinifera. Plant Physiol. 1966:41:923-31.

14. Moriguchi T, Abe K, Sanada T, Yamaki S. Levels and Role of sucrose synthase, sucrose-phosphate synthase, and acid invertase in sucrose accumulation in fruit of Asian pear. J Am Soc Hort Sci. 1992;117:274-8.

15. Moriguchi T, Ishizawa Y, Sanada T. Differences in sugar composition in Prunus persica fruit and the classification by the principal component analysis. J Jpn Soc Hort Sci. 1990;59:307-12.

16. Moriguchi T, Sanada T, Yamaki S. Seasonal fluctuations of some enzymes relating to sucrose and sorbitol metabolism in peach fruit. J Am Soc Hort Sci. 1990;115:278-81.

17. Yamaki S, Moriguchi T. Seasonal fluctuation of sorbitol-related enzymes and invertase activities accompanying maturation of Japanese Pear (Pyrus serotina Rehder var. culta Rehder) fruit. J Jpn Soc for Hortic Sci. 1989:57:602-7.

18. Doty T. Fructose sweetness: a new dimension. Cereal Foods World. 1976:21:62-3.

19. Pancoast HM, Junk WR. Handbook of sugars. Westport, Connecticut, USA: AVI Publishing Company; 1980. p. 387-9.

20. Pangborn R. Relative taste intensities of selected sugars and organic acids. J Food Sci. 1963;28:726-33.

21. Schneider K, Schäfer-Pregl R, Borchardt D, Salamini F. Mapping QTLs for sucrose content, yield and quality in a sugar beet population fingerprinted by EST-related markers. Theor Appl Genet. 2002;104:1107-13.

22. Ukoskit K, Posudsavang G, Pongsiripat N, Chatwachirawong P, Klomsa-Ard P, Poomipant $P$, et al. Detection and validation of EST-SSR markers associated with sugar-related traits in sugarcane using linkage and association mapping. Genomics. 2019;111:1-9.

23. Draffehn AM, Meller S, Li L, Gebhardt C. Natural diversity of potato (Solanum tuberosum) invertases. BMC Plant Biol. 2010;10:1-15. 
24. Kajiura I, Yamaki S, Omura M, Akihama T, Machida Y. Improvement of sugar content and composition in fruits, and classifications of East Asian pears by the principal component analysis of sugar compositions in fruits. Jpn J Breed. 1979;29:1-12.

25. Hecke K, Herbinger K, Veberic R, Trobec M, Toplak H, Stampar F, et al. Sugar-, acid- and phenol contents in apple cultivars from organic and integrated fruit cultivation. Eur J Clin Nutr. 2006;60:1136-40.

26. Wu J, Gao H, Zhao L, Liao X, Chen F, Wang Z, Hu X. Chemical compositional characterization of some apple cultivars. Food Chem. 2007;103:88-93.

27. Byrne DH, Nikolic AN, Burns EE. Variability in sugars, acids, firmness, and color characteristics of 12 peach genotypes. J Am Soc Hort Sci. 1991;116:1004-6.

28. Guan Y, Peace C, Rudell D, Verma S, Evans K. QTLs detected for individual sugars and soluble solids content in apple. Mol Breed. 2015;35:135.

29. Zhang RP, Wu J, Li XG, Khan MA, Chen H, Korban SS, et al. An AFLP, SRAP, and SSR genetic linkage map and identification of QTLs for fruit traits in Pear (Pyrus L.). Plant Mol Biol Rptr. 2013;31:678-87.

30. Li H, Huang C-H, Ma H. Whole-genome duplications in pear and apple. In: Korban SA, editor. The Pear Genome. New York: Springer; 2019. p. 279-99.

31. Sassa H. Molecular mechanism of the S-RNase-based gametophytic selfincompatibility in fruit trees of Rosaceae. Breeding Sci. 2016;66:116-21.

32. Moriya S, Terakami S, Okada K, Shimizu T, Adachi Y, Katayose Y, et al. Identification of candidate genes responsible for the susceptibility of apple (Malus $\times$ domestica Borkh.) to Alternaria blotch. BMC Plant Biol. 2019;19:132.

33. Terakami S, Moriya S, Adachi Y, Kunihisa M, Nishitani C, Saito T, et al. Fine mapping of the gene for susceptibility to black spot disease in Japanese pear (Pyrus pyrifolia Nakai). Breeding Sci. 2016;66:271-80.

34. Itai A, Kotaki T, Tanabe K, Tamura F, Kawaguchi D, Fukuda M. Rapid identification of 1-aminocyclopropane-1-carboxylate (ACC) synthase genotypes in cultivars of Japanese pear (Pyrus pyrifolia Nakai) using CAPS markers. Theor Appl Genet. 2003;106:1266-72.

35. Sato T, Kudo T, Akada T, Wakasa Y, Niizeki M, Harada T. Allelotype of a ripening-specific 1-aminocyclopropane-1-carboxylate synthase gene defines the rate of fruit drop in apple. J Am Soc Hort Sci. 2004;129:32-6.

36. Nishio S, Hayashi T, Yamamoto T, Yamada M, Takada N, Kato H, et al. Validation of molecular markers associated with fruit ripening day of Japanese pear (Pyrus pyrifolia Nakai) using variance components. Sci Hortic. 2016;199:9-14.

37. Kenis K, Keulemans J, Davey MW. Identification and stability of QTLs for fruit quality traits in apple. Tree Genet Genomes. 2008;4:647-61.

38. Kunihisa M, Moriya S, Abe K, Okada K, Haji T, Hayashi T, et al. Identification of QTLs for fruit quality traits in Japanese apples: QTLs for early ripening are tightly related to preharvest fruit drop. Breeding Sci. 2014;64:240-51.

39. Liebhard R, Kellerhals M, Pfammatter W, Jertmini M, Gessler C. Mapping quantitative physiological traits in apple (Malus $\times$ domestica Borkh.). Plant Mol Biol. 2003;52:511-26.

40. Cappai F, De Franceschi P, Ciriani A, Collina M, Dondini L. QTLs for susceptibility to Stemphylium vesicarium in pear. Mol Breed. 2018;38:24.

41. Daccord N, Celton J-M, Linsmith G, Becker C, Choisne N, Schijlen E, et al. High-quality de novo assembly of the apple genome and methylome dynamics of early fruit development. Nat Genet. 2017;49:1099.

42. Chagné $D$, Crowhurst RN, Pindo M, Thrimawithana A, Deng C, Ireland H, et al. The draft genome sequence of European pear (Pyrus communis $\mathrm{L}$. 'Bartlett'). PLoS One. 2014;9:e92644.

43. Wu J, Wang Z, Shi Z, Zhang S, Ming R, Zhu S, et al. The genome of the pear (Pyrus bretschneideri Rehd.). Genome Res. 2013;23:396-408.

44. Dong X, Wang Z, Tian L, Zhang Y, Qi D, Huo H, et al. De novo assembly of a wild pear (Pyrus betuleafolia) genome. Plant Biotechnol J. 2020;18:581-95.

45. Ishimizu T, Inoue K, Shimonaka M, Saito T, Terai O, Norioka S. PCR-based method for identifying the S-genotypes of Japanese pear cultivars. Theor Appl Genet. 1999;98:961-7.

46. Okada K, Tonaka N, Moriya Y, Norioka N, Sawamura Y, Matsumoto T, et al. Deletion of a $236 \mathrm{~kb}$ region around S4-RNase in a stylar-part mutant S4sm-haplotype of Japanese pear. Plant Mol Biol. 2008;66:389.

47. Terakami S, Adachi Y, Iketani H, Sato Y, Sawamura Y, Takada N, et al. Genetic mapping of genes for susceptibility to black spot disease in Japanese pears. Genome. 2007;50:735-41.
48. Terakami S, Shoda M, Adachi Y, Gonai T, Kasumi M, Sawamura Y, et al. Genetic mapping of the pear scab resistance gene $V n k$ of Japanese pear cultivar Kinchaku. Theor Appl Genet. 2006;113:743-52.

49. Shirasawa K, Hirakawa H, Isobe S. Analytical workflow of double-digest restriction site-associated DNA sequencing based on empirical and in silico optimization in tomato. DNA Res. 2016;23:145-53.

50. Meuwissen T, Hayes B, Goddard M. Prediction of total genetic value using genome-wide dense marker maps. Genetics. 2001;157:1819-29.

51. Hayashi T, Iwata H. A Bayesian method and its variational approximation for prediction of genomic breeding values in multiple traits. BMC Bioinformatics. 2013;14:34.

52. Saito T, Takada N, Kato H, Terakami S, Nishio S. Genotypic variation in and environmental variance components of sugar composition in Japanese pear fruit. HortScience. 2019;54:1465-9.

53. Abe K, Sato Y, Saito T, Kurihara A, Kotobuki K. Genetic correlation between ripening time and weight of fruits in Japanese pear (Pyrus pyrifolia Nakai). Jpn J Breed. 1993;43:439-47.

54. Moriya S, Kunihisa M, Okada K, Iwanami H, Iwata H, Minamikawa M, et al. Identification of QTLs for flesh mealiness in apple (Malus $\times$ domestica Borkh.). Hortic J. 2017:86:159-70.

55. Kumar S, Garrick DJ, Bink MC, Whitworth C, Chagné D, Volz RK. Novel genomic approaches unravel genetic architecture of complex traits in apple. BMC Genomics. 2013;14:393.

56. Leforestier D, Ravon E, Muranty H, Cornille A, Lemaire C, Giraud T, et al. Genomic basis of the differences between cider and dessert apple varieties. Evol Appl. 2015;8:650-61.

57. Saito T. Advances in Japanese pear breeding in Japan. Breeding Sci. 2016;66:46-59.

58. Kiyosue T, Abe H, Yamaguchi-Shinozaki K, Shinozaki K. ERD6, a cDNA clone for an early dehydration-induced gene of Arabidopsis, encodes a putative sugar transporter. Biochimica et Biophysica Acta (BBA)-Biomembranes. 1998;1370:187-91.

59. Poschet G, Hannich B, Raab S, Jungkunz I, Klemens PA, Krueger S, et al. A novel Arabidopsis vacuolar glucose exporter is involved in cellular sugar homeostasis and affects the composition of seed storage compounds. Plant Physiol. 2011;157:1664-76.

60. Afoufa-Bastien D, Medici A, Jeauffre J, Coutos-Thévenot P, Lemoine R, Atanassova R, Laloi M. The Vitis vinifera sugar transporter gene family: phylogenetic overview and macroarray expression profiling. BMC Plant Biol. 2010;10:245

61. Zhang C, Bian Y, Hou S, Li X. Sugar transport played a more important role than sugar biosynthesis in fruit sugar accumulation during Chinese jujube domestication. Planta. 2018;248:1187-99.

62. Shiratake K, Martinoia E. Transporters in fruit vacuoles. Plant Biotechnol. 2007;24:127-33.

63. Larsen B, Migicovsky Z, Jeppesen AA, Gardner KM, Toldam-Andersen TB, Myles $S$, et al. Genome-wide association studies in apple reveal loci for aroma volatiles, sugar composition, and harvest date. Plant Genome. 2019;12:180104.

64. Eduardo I, Picañol R, Rojas E, Batlle I, Howad W, Aranzana M, et al. Mapping of a major gene for the slow ripening character in peach: co-location with the maturity date gene and development of a candidate genebased diagnostic marker for its selection. Euphytica. 2015;205:627-36.

65. Pirona R, Eduardo I, Pacheco I, Linge CDS, Miculan M, Verde I, et al. Fine mapping and identification of a candidate gene for a major locus controlling maturity date in peach. BMC Plant Biol. 2013;13:166.

66. Bai Y, Dougherty L, Li M, Fazio G, Cheng L, Xu K. A natural mutation-led truncation in one of the two aluminum-activated malate transporter-like genes at the Ma locus is associated with low fruit acidity in apple. Mol Genet Genomics. 2012;287:663-78.

67. Verma S, Evans K, Guan Y, Luby JJ, Rosyara U, Howard NP, et al. Two largeeffect QTLS, Ma and Ma3, determine genetic potential for acidity in apple fruit: breeding insights from a multi-family study. Tree Genet Genomes. 2019;15:18.

68. Tamura F. Japanese pear. In: Sci JSH, editor. Horticulture in Japan 2006. Kyoto: Shoukadoh Publication; 2006. p. 50-8.

69. Kajiura I, Suzuki K, Yamazaki T. Color chart for Japanese pear (Pyrus serotina var. culta Rehder). HortScience. 1975;10:257-8.

70. Acquadro A, Barchi L, Gramazio P, Portis E, Vilanova S, Comino C, et al. Coding SNPs analysis highlights genetic relationships and evolution pattern in eggplant complexes. PLoS One. 2017;12:e0180774. 
71. Li H, Durbin R. Fast and accurate short read alignment with BurrowsWheeler transform. Bioinformatics. 2009;25:1754-60.

72. Li H, Handsaker B, Wysoker A, Fennell T, Ruan J, Homer N, et al. The sequence alignment/map format and SAMtools. Bioinformatics. 2009;25:2078-9.

73. Browning BL, Browning SR. Genotype imputation with millions of reference samples. Am J Hum Genet. 2016;98:116-26.

74. Alexander DH, Novembre J, Lange K. Fast model-based estimation of ancestry in unrelated individuals. Genome Res. 2009;19:1655-64.

75. Purcell S, Neale B, Todd-Brown K, Thomas L, Ferreira MA, Bender D, et al. PLINK: a tool set for whole-genome association and population-based linkage analyses. Am J Hum Genet. 2007;81:559-75.

76. Kopelman NM, Mayzel J, Jakobsson M, Rosenberg NA, Mayrose I. Clumpak: a program for identifying clustering modes and packaging population structure inferences across K. Mol Ecol Resour. 2015;15:1179-91.

77. Wickham H. Ggplot2: Elegant Graphics for Data Analysis. New York: Springer; 2009.
78. Endelman JB. Ridge regression and other kernels for genomic selection with R package rrBLUP. Plant Genome. 2011;4:250-5.

79. Nishio S, Hayashi T, Yamamoto T, Terakami S, Iwata H, Imai A, Takada N, Kato $\mathrm{H}$, Saito T. Bayesian genome-wide association study of nut traits in Japanese chestnut. Mol Breed. 2018;38:99.

80. Camacho C, Coulouris G, Avagyan V, Ma N, Papadopoulos J, Bealer K, et al. BLAST+: architecture and applications. BMC Bioinformatics. 2009;10:1-9.

\section{Publisher's Note}

Springer Nature remains neutral with regard to jurisdictional claims in published maps and institutional affiliations.
Ready to submit your research? Choose BMC and benefit from:

- fast, convenient online submission

- thorough peer review by experienced researchers in your field

- rapid publication on acceptance

- support for research data, including large and complex data types

- gold Open Access which fosters wider collaboration and increased citations

- maximum visibility for your research: over $100 \mathrm{M}$ website views per year

At BMC, research is always in progress.

Learn more biomedcentral.com/submissions 